\title{
Low dose of GRP78-targeting subtilase cytotoxin improves the efficacy of photodynamic therapy in vivo
}

\author{
MAGDALENA GABRYSIAK ${ }^{1}$, MALGORZATA WACHOWSKA ${ }^{1}$, JOANNA BARANKIEWICZ ${ }^{1,2,3}$, ZOFIA PILCH $^{1}$, \\ ANNA RATAJSKA ${ }^{4}$, EWA SKRZYPEK ${ }^{4}$, MAGDALENA WINIARSKA ${ }^{1}$, ANTONI DOMAGALA ${ }^{1}$, \\ TOMASZ P. RYGIEL ${ }^{1}$, ALICJA JOZKOWICZ ${ }^{5}$, LOUIS BOON ${ }^{6}$, JAKUB GOLAB $^{1 *}$ and MALGORZATA FIRCZUK ${ }^{*}$ \\ ${ }^{1}$ Department of Immunology, Center of Biostructure Research, Medical University of Warsaw, Banacha 1A, 02-097 Warsaw; \\ ${ }^{2}$ Department of Hematology and Transfusion Medicine, Centre of Postgraduate Medical Education, 01-813 Warsaw; \\ ${ }^{3}$ Department of Hematology, Institute of Hematology and Transfusion Medicine, 02-776 Warsaw; \\ ${ }^{4}$ Department of Pathology, Center of Biostructure Research, Medical University of Warsaw, 02-004 Warsaw; \\ ${ }^{5}$ Department of Medical Biotechnology, Faculty of Biochemistry, Biophysics and Biotechnology, Jagiellonian University, \\ 30-387 Kracow, Poland; ${ }^{6}$ EPIRUS Biopharmaceuticals Netherlands BV, 3584 CM Utrecht, The Netherlands
}

Received November 19, 2015; Accepted December 23, 2015

DOI: $10.3892 /$ or.2016.4723

\begin{abstract}
Photodynamic therapy (PDT) exerts direct cytotoxic effects on tumor cells, destroys tumor blood and lymphatic vessels and induces local inflammation. Although PDT triggers the release of immunogenic antigens from tumor cells, the degree of immune stimulation is regimen-dependent. The highest immunogenicity is achieved at sub-lethal doses, which at the same time trigger cytoprotective responses, that include increased expression of glucose-regulated protein 78 (GRP78). To mitigate the cytoprotective effects of GRP78 and preserve the immunoregulatory activity of PDT, we investigated the in vivo efficacy of PDT in combination with EGF-SubA cytotoxin that was shown to potentiate in vitro PDT cytotoxicity by inactivating GRP78. Treatment of immunocompetent BALB/c mice with EGF-SubA improved the efficacy of PDT but only when mice were treated with a dose of EGF-SubA that exerted less pronounced effects on the number of $\mathrm{T}$ and $\mathrm{B}$ lymphocytes as well as dendritic cells in mouse spleens. The observed antitumor effects were critically dependent on $\mathrm{CD} 8^{+} \mathrm{T}$ cells and were completely abrogated in immunodeficient SCID mice. All these results suggest that GRP78 targeting improves in vivo PDT efficacy provided intact T-cell immune system.
\end{abstract}

Correspondence to: Dr Malgorzata Firczuk, Department of Immunology, Center of Biostructure Research, Medical University of Warsaw, la Banacha Street, F building, 02-097 Warsaw, Poland E-mail:mfirczuk@wum.edu.pl

\section{*Contributed equally}

Key words: cancer, photodynamic therapy, immune response, T cell, EGF-SubA

\section{Introduction}

Photodynamic therapy (PDT) is an approved anticancer treatment, which involves systemic or local administration of a photosensitizer (PS) followed by illumination of the tumor with visible light. In the presence of oxygen it generates reactive oxygen species (ROS), which exert direct cytotoxic effects on tumor cells, as well as on the tumor vasculature (1). PDT leads to a significant decrease or elimination of the primary tumor. However, for long-term efficacy of PDT, the intact immune response is essential (2). It was demonstrated that in immunocompromised animals devoid of adaptive immunity, PDT initially triggers tumor ablation, but permanent cures are not observed (3). Specific depletion of selected populations of leukocytes revealed that neutrophils and $\mathrm{CD} 8^{+} \mathrm{T}$ cells play an indispensable role in achieving maximum therapeutic effects of PDT (4). Further studies confirmed that PDT generates damage-associated molecular pattern (DAMP) proteins and that PDT-triggered cell death is associated with immunogenic antigens $(5,6)$. However, induction of the immune response by PDT depends primarily on the treatment regimen as well as on the tumor model. It was demonstrated that low-dose PDT stimulates an inflammatory response better than high-dose PDT, both in mice (7) and in clinical settings (8). A number of approaches to enhance the immune response triggered by PDT have been proposed, including combinations with immunestimulating agents $(9,10)$.

On the other hand, the sub-lethal PDT doses, optimal for immune stimulation, are usually insufficient to eradicate primary tumor due to induction of cytoprotective responses. To counterbalance PDT-triggered oxidative stress, tumor cells upregulate ROS-scavenging enzymes, stress-related transcription factors and chaperones. Moreover, the accumulation of misfolded proteins in the endoplasmic reticulum (ER) triggers unfolded protein response (UPR). It was shown that PDT induces expression of the master regulator of the UPR, glucose regulated protein 78 (GRP78) $(11,12)$. GRP78 is an 
endoplasmic reticulum resident chaperone, which is highly expressed in tumor cells. Increasing number of studies reveals that this protein is upregulated in response to various therapies and contributes to treatment resistance (13). Therefore, attempts to inhibit GRP78 are considered as components of anticancer therapy. In contrast to several small-molecule GRP78 inhibitors, which are very unspecific, a bacterial subtilase cytotoxin (Sub) has been shown to specifically cleave and inactivate GRP78 (14). The holotoxin (SubAB5) produced by Shiga toxigenic strains of Escherichia coli (STEC), is composed of a catalytic A subunit (SubA) and a pentameric B subunit, enabling cell entry. Previous research has revealed that the toxin is the major cause of hemolytic uremic syndrome, the most common form of the disease observed in humans infected with STEC. It is also lethal in mice, as it triggers substantial systemic abnormalities due to microvascular damage, thrombosis and hemorrhage in several organs, including the kidneys, brain, and liver (15). Further study has shown that the toxin also affects the immune system, which is manifested by spleen atrophy and profound leukocyte redistribution (16). Moreover, sub-lethal doses of SubAB5 exert anti-inflammatory effects (17). To exploit the antitumor activity of the toxin via GRP78 targeting, as well as to minimize severe toxic effects, the fusion protein composed of epidermal growth factor (EGF) and the catalytic A subunit of the toxin (EGF-SubA) was generated for targeted toxin delivery. EGF-SubA selectively killed EGF receptor (EGFR)-expressing tumor cells and had an antitumor efficacy in a mouse model. Moreover, EGF-SubA synergized with UPR-inducing agents such as tunicamycin or thapsigargin (18).

We recently found that the expression of GRP78 was induced in vitro by Photofrin-PDT in human cancer cell lines and that GRP78 plays a cytoprotective role. Moreover, siRNA-mediated downregulation of GRP78 as well as EGF-SubA-mediated specific cleavage of GRP78, sensitized tumor cells to in vitro PDT (19). Here, we investigated the combination of EGF-SubA with PDT in vivo in mouse models.

\section{Materials and methods}

Reagents. Photofrin (Axcan Pharma Inc., Houdan, France) was used as a photosensitizer. Photofrin was dissolved in $5 \%$ glucose, aliquoted and stored at $-20^{\circ} \mathrm{C}$. EGF-SubA fusion protein was purchased from SibTech Inc. (Brookfield, CT, USA). The $0.75 \mu \mathrm{M}$ (in vitro studies) or $1 \mathrm{mg} / \mathrm{ml}$ (in vivo studies) stock solutions were aliquoted and stored at $-20^{\circ} \mathrm{C}$.

Cell culture. Mouse colon carcinoma (CT26) and human prostate cancer (DU-145) cell lines were purchased from the American Type Culture Collection (ATCC; Rockville, MD, USA). Cells were cultured in Roswell Park Memorial Institute (RPMI)-1640 medium (CT26) or Dulbecco's modified Eagle's medium (DMEM) (DU-145) supplemented with 20\% (CT26) or 5\% (DU-145) heat-inactivated fetal bovine serum (Hyclone) and antibiotic/antimycotic solution (Sigma-Aldrich, St. Louis, MO, USA). Cells were cultured under standard conditions in a $5 \% \mathrm{CO}_{2}$ humidified incubator at $37^{\circ} \mathrm{C}$.

Generation of CT26 cells stably expressing human EGFR. A CT26 cell line stably expressing human EGFR (CT26-EGFR) was generated using a retroviral system. HEK 293T cells were co-transfected with the pBabe-EGFR-puro plasmid, packaging pKAT and envelope pVSV-G vectors using a calcium chloride protocol. The pBabe-EGFR-puro plasmid encoding WT human EGFR was a gift from Matthew Meyerson (plasmid \#11011; Addgene) (20). Twenty-four hours post-transfection, medium from the HEK 293 T cells was collected, centrifuged and filtered through a $0.45-\mu \mathrm{m}$ filter. Afterwards, the CT26 cells were added to retrovirus-containing medium and centrifuged for $1 \mathrm{~h}(500 \mathrm{x} \mathrm{g}, \mathrm{RT})$ in the presence of $12 \mu \mathrm{g} /$ $\mathrm{ml}$ Polybrene (Sigma-Aldrich). Next, the cells were selected with $10 \mu \mathrm{g} / \mathrm{ml}$ puromycin (Sigma-Aldrich) and sorted for high EGFR expression with cell sorter FACSAria III (Becton Dickinson). The overexpression of EGFR was confirmed by flow cytometry and western blotting.

Cell viability assay. In order to evaluate the cytostatic/cytotoxic effect of EGF-SubA on cancer cells, $1.25 \times 10^{3}$ cells/well were plated onto 96 -well plates, incubated with increasing concentrations of EGF-SubA for $48 \mathrm{~h}$ and stained with crystal violet as described previously (21).

Flow cytometry and cell sorting. To confirm the presence of EGFR on the cell surface, the cells were trypsinized, pelleted, washed with PBS and blocked in 1\% BSA for 15 min. Next, the cells were incubated with chimeric anti-EGFR antibody (Erbitux) for 40 min (1:50) followed by incubation with anti-human IgG-Alexa488 antibody (1:100, 30 min; Jackson ImmunoResearch). The cells were analyzed by FACScan (Becton Dickinson) using CellQuest Pro software version 5.2. To select the EGFR-expressing population, the CT26 cells were stained for surface EGFR as described and Alexa488positive cells were sorted for using FACSAria III (Becton Dickinson).

Splenocyte isolation and analysis of leukocyte populations. Spleens were isolated from mice of each experimental group. To obtain a single-cell suspension, the spleens were forced through a $70-\mu \mathrm{m}$ cell strainer. To lyse erythrocytes, the cells were incubated for $5 \mathrm{~min}$ at $37^{\circ} \mathrm{C}$ in red blood cell lysis buffer (150 $\mathrm{mM} \mathrm{NH}_{4} \mathrm{Cl}, 1 \mathrm{mM} \mathrm{NaHCO}_{3}, \mathrm{pH}$ 7.4). Next, the cells were washed with PBS and resuspended in RPMI-1640 medium containing $10 \%$ fetal bovine serum.

To determine the total splenocyte count, the cells were stained with anti-CD45 antibody and the number of viable cells in $10 \mu \mathrm{l}$ was determined using the Accuri C6 flow cytometer (Becton Dickinson). To evaluate leukocyte populations, the splenocytes were stained with the following monoclonal antibodies (mAbs): anti-B220-eFluor ${ }^{\circledR} 450$ (REF. 48-0452-80), anti-CD3-PE-Cy7 (REF. 25-0031-82), antiCD4-APC (REF. 17-0041), anti-CD8-FITC (REF. 11-0081), anti-CD11c-eFluor ${ }^{\circledR} 450$ (REF. 48-0114) (all from eBioscience) anti-CD45.2-BD HorizonBD Horizon ${ }^{\mathrm{TM}}$ V500 (REF. 562129; BD Biosciences), anti-MHCII-PE-Cy7 (REF. 25-5321; eBioscience) and analyzed using FACSAria III.

Western blotting. Cells were pelleted and lysed in a lysis buffer (50 mM HEPES pH 7.4, 1.0\% Triton X-100, 150 mM NaCl, $10 \%$ glycerol, 5 mM EDTA) supplemented with Complete protease inhibitors (Roche, Mannheim, Germany) and phos- 
phatase inhibitors $(1 \mathrm{mM}$ sodium ortho-vanadate, $1 \mathrm{mM}$ sodium fluoride, and $1 \mathrm{mM} 2$-glycerol phosphate). The protein concentration was measured using the Bio-Rad protein assay (Bio-Rad, Hercules, CA, USA). Equal amounts of proteins were separated by SDS-PAGE and transferred to Protran ${ }^{\circledR}$ nitrocellulose membranes (Schleicher and Schuell BioScience, Dassel, Germany). The membranes were blocked and incubated with the following primary antibodies (GRP78-3183; Cell Signaling Technology, Beverly, MA, USA; $\beta$-actin-A3854; SigmaAldrich) according to the manufacturer's protocols. After extensive washing with TBST, the membranes were incubated for 40 min with HRP-linked secondary antibodies (Cell Signaling Technology). The chemiluminescence reaction was developed using self-made reagent $(50 \mathrm{mM}$ Tris- $\mathrm{HCl} \mathrm{pH} 8.5$, $0.2 \mathrm{mM}$ cumaric acid, $1.25 \mathrm{mM}$ luminol, $0.006 \%$ hydrogen peroxide) and visualized with Stella 8300 Bio-imager (Raytest, Straubenhardt, Germany).

Mice. For the in vivo experiments 8- to 14-week-old female $\mathrm{BALB} / \mathrm{c}$ or SCID mice were used. BALB/c mice were obtained from the Animal House of the Polish Academy of Sciences, Medical Research Center (Warsaw, Poland). SCID mice were obtained from Charles River Laboratory (Erkrath, Germany). The experiments were performed in accordance with the guidelines approved by the Ethics Committee of the Medical University of Warsaw.

PDT in vivo. Before inoculation, the cells were harvested, washed twice and resuspended with PBS (CT26-EGFR) or PBS with 25\% Matrigel (DU-145). The viability of the cells was determined by trypan blue staining. A total of $3 \times 10^{5}$ CT26EGFR cells (in $30 \mu \mathrm{l}$ ) or $2 \times 10^{6}$ of DU-145 cells (in $100 \mu \mathrm{l}$ ) were injected subcutaneously into the right thigh of the experimental mice (day 0). On days 6-8 (BALB/c) or 7-9, the (SCID) mice were injected intraperitoneally (i.p.) with EGF-SubA (25 or $50 \mu \mathrm{g} / \mathrm{kg}$ ) in PBS. Photofrin was administered i.p. at a dose of $10 \mathrm{mg} / \mathrm{kg}$ on day 6 (BALB/c) or day 7 (SCID). Twenty-four hours later, the tumors were illuminated with $630 \mathrm{~nm}$ light delivered by a He-Ne ion laser (Laserinstruments, Warsaw, Poland) through optical fibre. The power of the laser was $45-47 \mathrm{~mW} / \mathrm{cm}^{2}$ and the total fluence was $50 \mathrm{~J} / \mathrm{cm}^{2}$ for CT26 and $43 \mathrm{~J} / \mathrm{cm}^{2}$ for DU-145 cells. During the time of illumination, the mice were anesthetized with ketamine and xylazine and restrained. Tumor growth was monitored 3 times a week (BALB/c) or once a week (SCID) with the use of a caliper, as described previously (22). The mice were sacrificed when any of the tumor diameters reached $15 \mathrm{~mm}$.

Depletion. Mice were injected i.p. with $100 \mu \mathrm{g}$ of anti-CD8 (YTS169) monoclonal antibodies (mAbs) on days 5 and 11 of the experiment. Control mice received $100 \mu \mathrm{g}$ of isotype control mAb. The level of depletion was evaluated 3 days after $\mathrm{mAb}$ injection. Blood samples were collected from the jugular vein of the experimental mice, stained with the anti-CD8 mAb and analyzed by flow cytometry.

Histopathologic analysis. After animal sacrifice, livers, spleens and kidneys were removed and fixed in buffered $4 \%$ formaldehyde for $48 \mathrm{~h}$ at RT. Subsequently, the tissue samples were dehydrated through a series of graded ethanol baths to
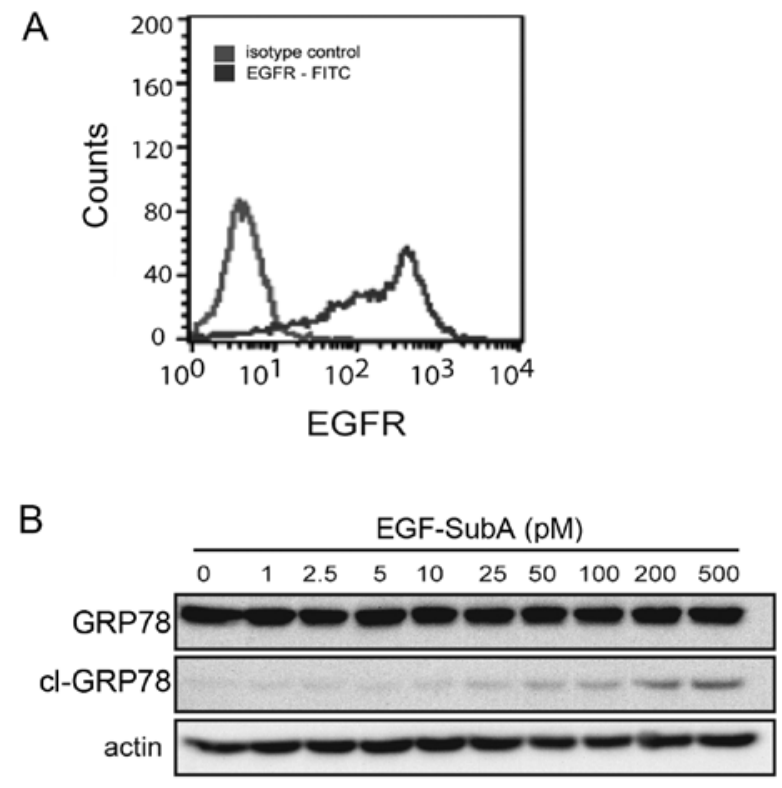

C
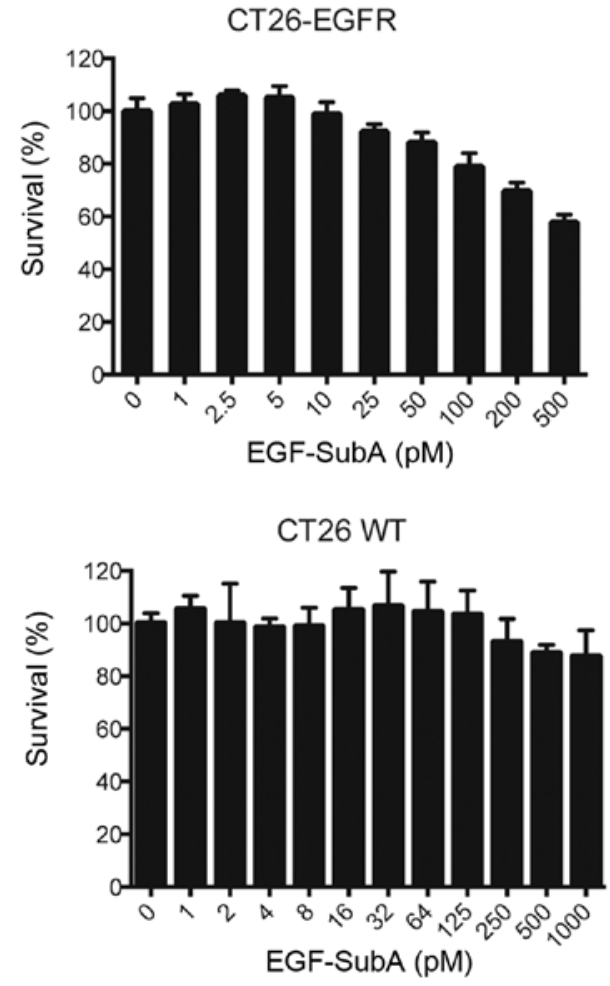

Figure 1. EGF-SubA exerts cytostatic/cytotoxic effects on CT26 cells expressing human EGFR. (A) CT26 cells were infected with retroviruses containing human EGFR and sorted for high expression of surface EGFR. The level of expression was confirmed by flow cytometry. (B) CT26-EGFR cells were plated onto $60-\mathrm{mm}$ dishes and incubated for $48 \mathrm{~h}$ with different concentrations of EGF-SubA. The amounts of GRP78 protein and the 46-kDa GRP78 cleavage product (cl-GRP78) were evaluated by western blotting. Actin was used as a loading control. (C) CT26-EGFR and CT26 WT cells were plated onto a 96-well plate and incubated for $48 \mathrm{~h}$ with increasing concentrations of EGF-SubA. The viability of the cells was determined by crystal violet staining.

displace the water, cleared in xylene, infiltrated with paraffin wax and then embedded in paraffin blocks. Paraffin sections 
A

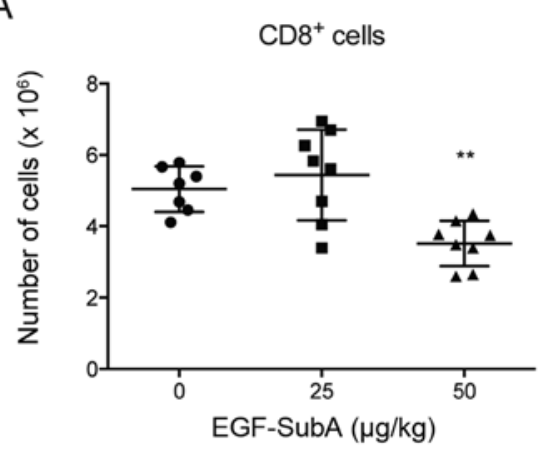

C

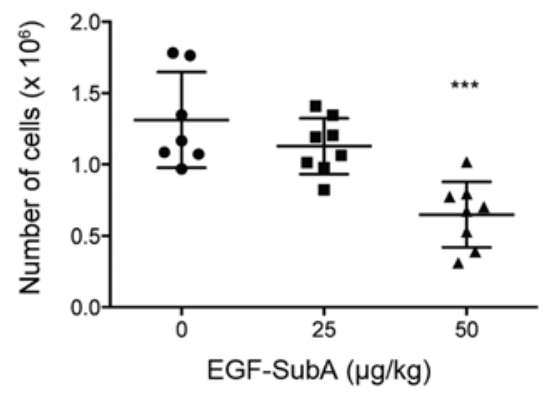

$\mathrm{E}$

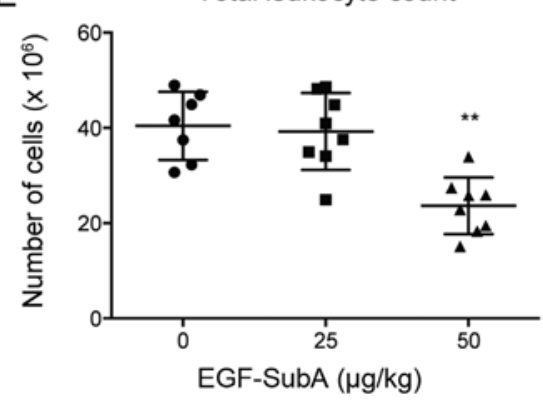

B

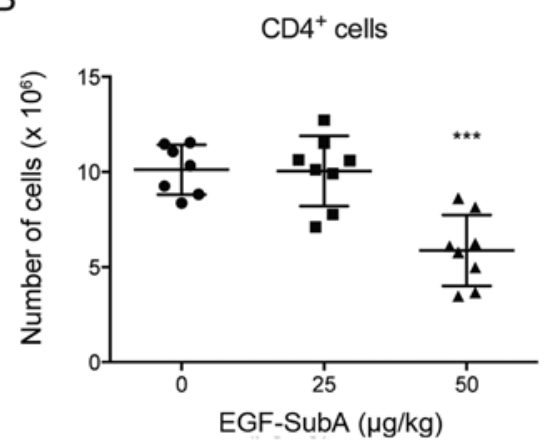

$\mathrm{D}$

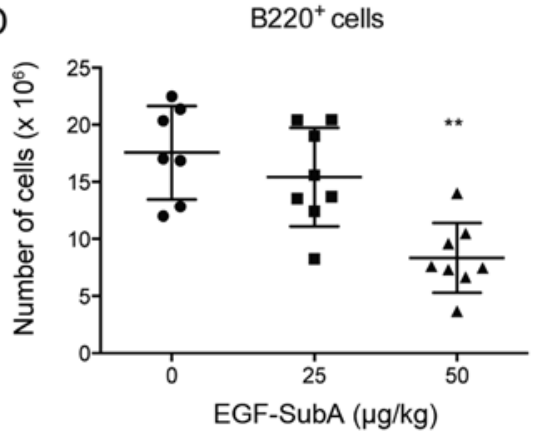

Figure 2. EGF-SubA affects immune cells. EGF-SubA at a dose of 25 or $50 \mu \mathrm{g} / \mathrm{kg}$ was administered i.p. for three consecutive days. Twenty-four hours after the last injection, mouse spleens were collected, total numbers of splenocytes were counted and analyzed by flow cytometry. Total numbers of cytotoxic (A) and helper (B) T cells, dendritic cells (C), B cells (D), and total leukocytes (E) were calculated. $\mathrm{n}=7$ (control group), $\mathrm{n}=8$ (25 and $50 \mu \mathrm{g}$ ) $\mathrm{kg}) .{ }^{*} \mathrm{P}<0.05,{ }^{* *} \mathrm{P}<0.01,{ }^{* * *} \mathrm{P}<0.005$ compared with the control group (MannWhitney test). were subsequently stained with hematoxylin and eosin (H\&E). All tissue sections were examined using light microscopy.

Statistical analyses. Differences in tumor volumes were analyzed for significance by the Mann-Whitney test with significance level set at $\mathrm{P}<0.05$. For ex vivo studies significance was calculated by the Mann-Whitney test. The survival rate of the animals was analyzed for significance by log-rank survival analysis. Significance was defined as a two-sided $\mathrm{P}<0.05$.

\section{Results}

EGF-SubA exerts cytostatic/cytotoxic effects on CT26 cells expressing human EGFR. We previously observed that EGF-SubA potentiated the cytotoxic effects of PDT in various human cancer cell lines in vitro (19). In order to study the effects of a combination of PDT and EGF-SubA in vivo, we generated a murine cell line CT26 expressing human EGFR (CT26EGFR). The presence of EGFR on the surface of the CT26 cells was confirmed by flow cytometry (Fig. 1A). To determine the efficacy of EGF-SubA, CT26-EGFR cells were incubated with increasing concentrations of the cytotoxin and tested for its ability to cleave GRP78 and to exert cytostatic/cytotoxic effects. Treatment with EGF-SubA resulted in accumulation of the GRP78 cleavage product (Fig. 1B). Moreover, the expression of EGFR sensitized CT26 cells to EGF-SubA as shown in the crystal violet cell viability assay (Fig. 1C).

Effects of EGF-SubA on murine immune cells and organs. Considering that an intact immune system is essential for PDT efficacy and that previous studies have described the impact of the SubAB5 holotoxin on immune cells, we investigated whether similar effects could be observed in mice treated with EGF-SubA. BALB/c mice were treated with 25 or $50 \mu \mathrm{g} / \mathrm{kg}$ of EGF-SubA for three consecutive days. Twenty-four hours after the last dose administration, spleens were collected, total numbers of splenocytes were counted and cells were analyzed by flow cytometry. We observed that a lower dose of EGF-SubA did not affect the immune cells, whereas the higher dose significantly reduced the numbers of B cells $\left(\mathrm{B} 220^{+}\right)$ and cytotoxic $\left(\mathrm{CD}^{+}\right)$and helper $\left(\mathrm{CD}^{+}\right) \mathrm{T}$ cells, as well as dendritic cells $\left(\mathrm{MHCII}^{+} \mathrm{CD} 11 \mathrm{c}^{+}\right.$) (Fig. 2).

Moreover, livers, spleens and kidneys were removed after 1, 2 and 3 doses of EGF-SubA and analyzed histopathologically. In mice treated with $25 \mu \mathrm{g} / \mathrm{kg}$ EGF-SubA, we occasionally observed hemorrhagic foci and hepatic necrosis as well as 


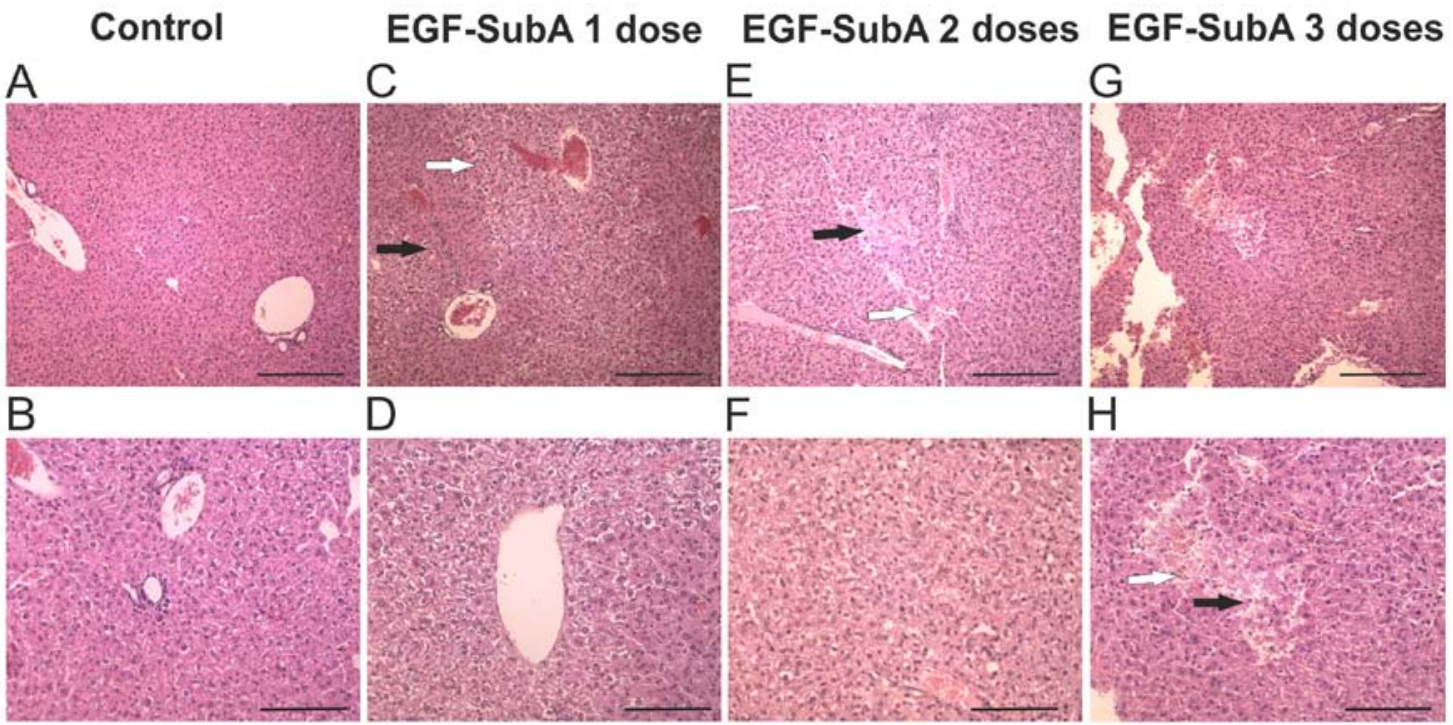

Figure 3. EGF-SubA causes pathological changes in murine livers. Hematoxylin and eosin-stained liver sections from control and $50 \mu \mathrm{g} / \mathrm{kg}$ EGF-SubA-treated mice, scale bars A, C, E and G $=250 \mu \mathrm{m} ; \mathrm{B}, \mathrm{D}, \mathrm{F}$ and $\mathrm{H}=125 \mu \mathrm{m}$. (A and B) Periportal area (centrilobular area) of control liver. (C) Hepatocytes with various densities of cytoplasm; the white arrow indicates the area around a central vein; the black arrow indicates the periportal area. (D) A higher magnification of the area around a central vein. (E) An area of hemorrhagic foci and necrosis are indicated with white and black arrows, respectively. (F) Dispersed lymphocytic infiltration. (G and $\mathrm{H})$ Necrosis with erythrocytes, marked with arrows.

very subtle changes in spleens and kidneys (data not shown). More pronounced changes were observed in the mice treated with $50 \mu \mathrm{g} / \mathrm{kg}$ of EGF-SubA. Splenic abnormalities included small accumulations of reticular fibers in the white pulp; renal abnormalities ranged from subtle edematous changes in deep cortical layer tubules to more pronounced focal deposits of eosinophilic material in some tubules, with obliteration of their lumina. The most noticeable changes we observed in the livers of mice treated with $50 \mu \mathrm{g} / \mathrm{kg}$ of EGF-SubA (Fig. 3). After one or two doses of the toxin we observed pronounced hepatocyte anisocytosis and occasional hemorrhagic foci. Hepatocytes located adjacent to the periportal areas were denser, whereas those located around central veins were edematous with pale cytoplasm. After three doses of EGF-SubA, vesicular fatty changes predominated in the cytoplasm of hepatocytes located in centrilobular areas. Moreover, isolated foci of necrosis and hemorrhagic changes were also visible with occasional areas of hepatocyte anisocytosis. Altogether, we observed that a $50 \mu \mathrm{g} / \mathrm{kg}$ dose of EGF-SubA negatively affected the immune cells and triggered histopathological changes.

EGF-SubA improves PDT efficacy. To determine whether EGF-SubA is able to potentiate the antitumor effects of PDT in vivo, we used the CT26-EGFR cell line. BALB/c mice subcutaneously injected with the CT26-EGFR cells were treated with PDT on day 7 after inoculation of tumor cells and EGF-SubA was administered at a dose of $25 \mu \mathrm{g} / \mathrm{kg}$ for three consecutive days (days 6-8) (Fig. 4A). We observed that the combination treatment resulted in a trend towards decreased tumor size (Fig. 4B) as well as prolonged survival (Fig. 4C), as compared with PDT alone. The difference in survival of mice treated with PDT only and PDT in combination with EGF-SubA was noticeable, and close to statistical significance $(\mathrm{P}=0.067, \log$-rank test). These effects were not observed in the mice treated with a higher dose $(50 \mu \mathrm{g} / \mathrm{kg})$ of EGF-SubA (Fig. 4D and E).
A functional immune system is necessary for the effects of the combination treatment. All the above data suggest that a functional immune response is essential for the final outcome of the combination treatment. In order to confirm this hypothesis, we performed the combination treatment in mice depleted of $\mathrm{CD}^{+}$cells, which have been shown essential for immune effects of PDT (Fig. 5A) (4). Depletion of CD8 ${ }^{+}$cells in the BALB/c mice completely abrogated the effects of the combination treatment on tumor size (Fig. 5B). Furthermore, no complete responses were observed in the $\mathrm{CD} 8^{+}$-depleted mice (Fig. 5C).

To further confirm that the functional immune system is crucial for the efficacy of PDT + EGF-SubA treatment, we aimed to determine whether the combination could be effective in immunocompromised mice. Previously, we observed a strong potentiation of in vitro cytostatic/cytotoxic effects of PDT in the human prostate cancer cell line DU-145 (19). Therefore, SCID mice were subcutaneously injected with DU-145 cells and treated with PDT on day 8 after inoculation of tumor cells and with EGF-SubA at a dose of $25 \mu \mathrm{g} / \mathrm{kg}$ for three consecutive days (days 7-9) (Fig. 6A). Treatment of DU-145 tumors with PDT resulted in the prolonged survival of SCID mice, however, no complete responses were observed. Despite the initial mild effects on tumor growth (Fig. 6B), the addition of EGF-SubA to the PDT protocol did not influence mouse survival in this model (Fig. 6C).

\section{Discussion}

The clinical application of PDT in cancer treatment is still limited to a small number of indications. However, the undeniable feature of PDT is its potential to elicit a specific antitumor immune response. Importantly, the ability of local PDT to induce systemic antitumor immunity has been demonstrated in patients with basal cell carcinoma (8). However, the degree of immune response induction by PDT is variable, regimen- 


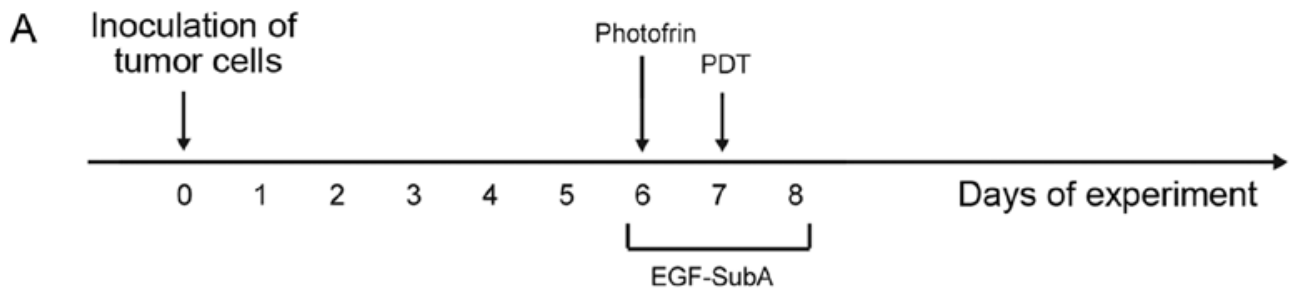

B

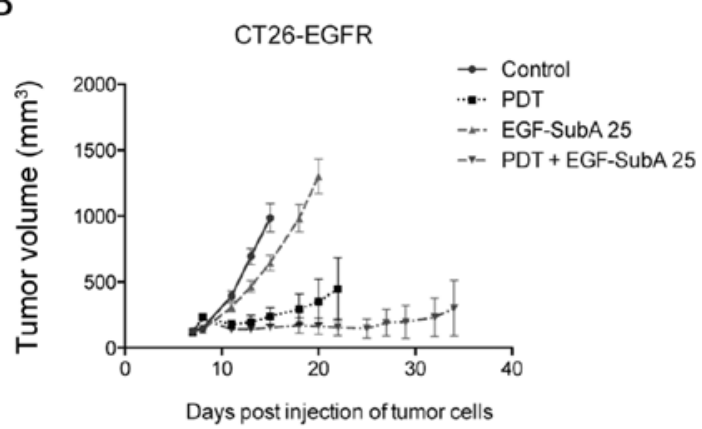

D

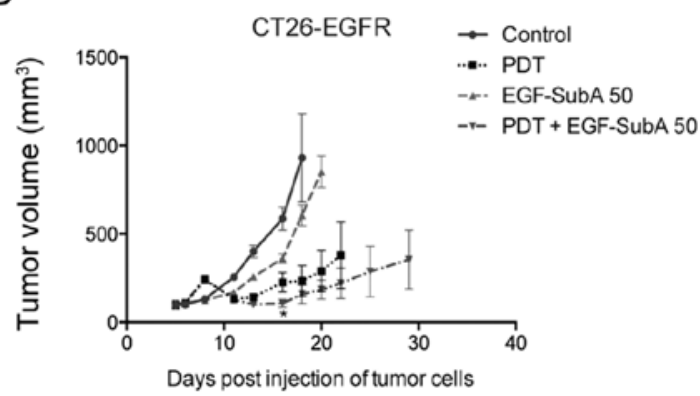

C

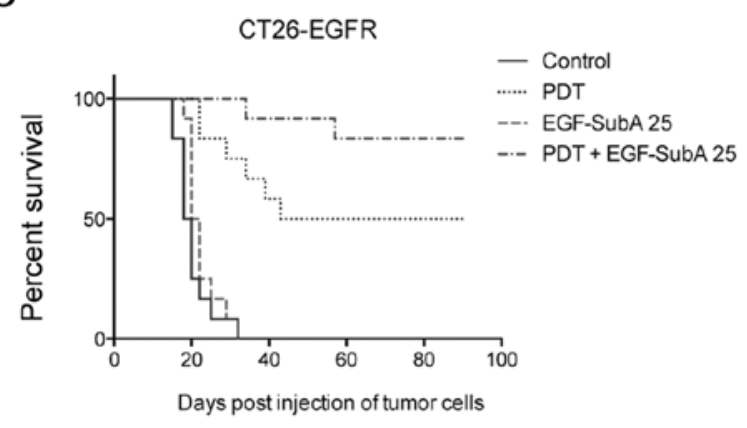

E

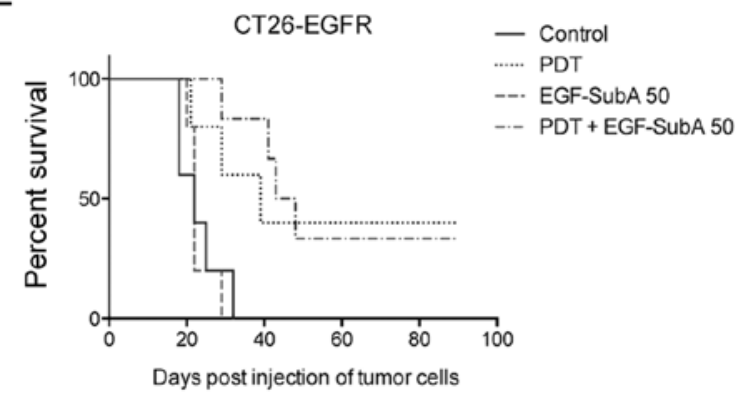

Figure 4. EGF-SubA at a dose of $25 \mu \mathrm{g} / \mathrm{kg}$ potentiates the antitumor effects of PDT in BALB/c mice injected with CT26-EGFR cells. (A) Mice were inoculated with $3 \times 10^{5}$ CT26-EGFR cells. On days 6-8 mice were treated with EGF-SubA at a dose of $25 \mu \mathrm{g} / \mathrm{kg}$ (B and C) or $50 \mu \mathrm{g} / \mathrm{kg}$ (D and E). Photofrin was administered i.p. at a dose of $10 \mathrm{mg} / \mathrm{kg}$ on day 6 . Twenty-four hours later, the tumor site was illuminated with laser light at a fluence of $50 \mathrm{~J} / \mathrm{cm}^{2}$. (B and D) Mean tumor volumes $( \pm \mathrm{SE})$. Experiments were performed twice (EGF-SubA $25 \mu \mathrm{g} / \mathrm{kg}$ ) or once (EGF-SubA $50 \mu \mathrm{g} / \mathrm{kg}$ ). One representative result is shown for EGF-SubA $25 \mu \mathrm{g} / \mathrm{kg}$ ( $\mathrm{n}=7-8$ for all experimental groups). (C and E) Kaplan-Meyer plots of the survival of the mice bearing CT26-EGFR tumors. The overall survival from two combined independent experiments is shown for EGF-SubA $25 \mu \mathrm{g} / \mathrm{kg}(\mathrm{n}=12)$. P=0.067 (PDT + EGF-SubA compared to all other groups, log-rank test). $\mathrm{n}=5-6$ in all experimental groups in the EGF-SubA $50 \mu \mathrm{g} / \mathrm{kg}$ group.

dependent and in the majority of models insufficient to cure cancer. Paradoxically, the most immune-stimulatory regimens are concomitantly sub-cytotoxic and the overall PDT effect is insufficient to obtain long-term responses. Therefore, the continuous search for treatment modalities combining PDT with other antitumor agents is warranted. Notably, our previous in vitro study in human cancer cell lines revealed a strong synergism between PDT and EGF-SubA cytotoxin (19).

Here, we showed that a low dose of EGF-SubA, the cytotoxin that cleaves and inactivates the master ER chaperone GRP78, increased the efficacy of PDT in vivo in immunocompetent mice. However, the antitumor effects were critically dependent on a functional immune response. Firstly, the antitumor effects were observed for the low $(25 \mu \mathrm{g} / \mathrm{kg})$, and not for the high $(50 \mu \mathrm{g} / \mathrm{kg})$ EGF-SubA dose (Fig. 4). Administration of the latter strongly depleted immune cells such as $\mathrm{CD} 8^{+}$and $\mathrm{CD} 4^{+} \mathrm{T}$ cells, $\mathrm{B}$ cells and dendritic cells in the spleens of mice. These effects were not observed in animals treated with the low EGF-SubA dose (Fig. 2). Secondly, the depletion of $\mathrm{CD}^{+} \mathrm{T}$ cells with monoclonal antibodies entirely abolished the antitumor effects of the combination treatment on both tumor growth rate and animal survival, supporting the fundamental contribution of these cells to the treatment outcome (Fig. 5B and C). Finally, in immunocompromised mice devoid of adaptive immunity, although the tumor-suppressive effect of both PDT alone as well as PDT with EGF-SubA were evident in a few days following PDT, none of the therapies were effective in the long run (Fig. 6B and C). All these results demonstrated that the combination of PDT with EGF-SubA may be effective providing an intact immune response.

Our results support previous findings that the immune response is crucial for overall PDT efficacy $(3,23)$. The role of selected immune cell populations in the PDT-induced antitumor immune response was extensively studied in various experimental models over the last two decades. Our results, as the majority of other reports $(4,10,24)$, substantiate the role of $\mathrm{CD}^{+} \mathrm{T}$ cells as critically important players in the maintenance of durable PDT-induced curative effects. Moreover, we observed that both the depletion of $\mathrm{CD}^{+} \mathrm{T}$ cells (Fig. 5) as well as the decrease in a number of $\mathrm{CD}^{+} \mathrm{T}$ cells and other immune cells in the spleens of mice treated with a higher dose of EGF-SubA (Fig. 2), negatively affected PDT efficacy. These 

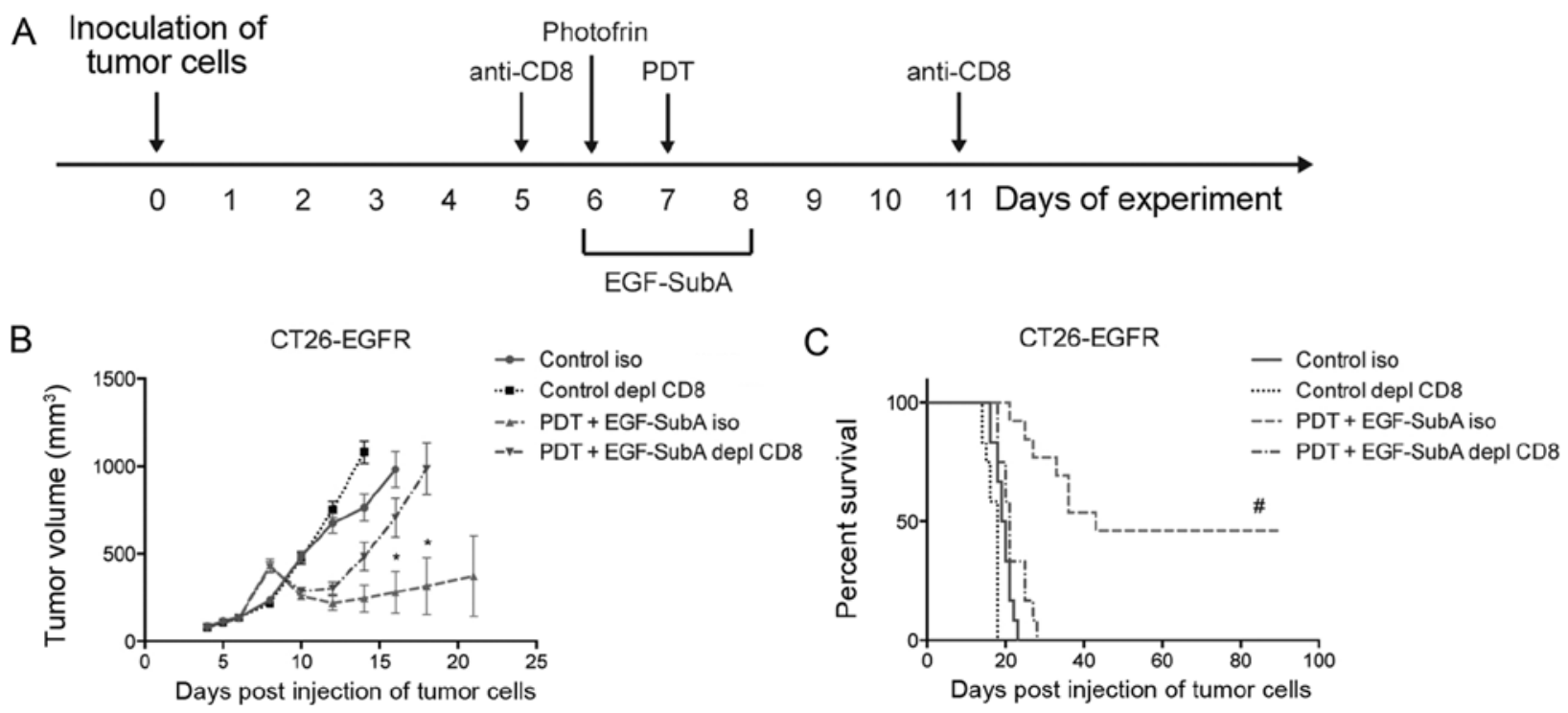

Figure 5. The efficacy of the combination treatment is dependent on CD8 ${ }^{+} \mathrm{T}$ cells. (A) BALB/c mice were inoculated with $3 \times 10^{5}$ CT26-EGFR cells. On days 6-8, the mice were treated with EGF-SubA at a dose of $25 \mu \mathrm{g} / \mathrm{kg}$. Photofrin was administered i.p. at a dose of $10 \mathrm{mg} / \mathrm{kg}$ on day 6 . Twenty-four hours later, the tumor site was illuminated with laser light at fluence of $50 \mathrm{~J} / \mathrm{cm}^{2}$. On days 5 and 11, the mice were injected i.p. with $100 \mu \mathrm{g}$ of anti-CD8 (YTS169) $\mathrm{mAbs}$ or a control antibody. (B) Mean tumor volumes $( \pm$ SE). The experiment was repeated twice and a representative result is shown. Depl (depletion) describes group of mice treated with depleting $\mathrm{mAbs}$ and iso refers to the group treated with isotype control mAbs ( $\mathrm{n}=6$ for control iso and control depl, $\mathrm{n}=5$ for PDT $+\mathrm{EGF}$ SubA iso and $n=8$ for PDT + EGF-SubA depl). (C) Kaplan-Meyer plots of the survival of the mice bearing CT26-EGFR tumors. The overall survival from two combined independent experiments is shown ( $\mathrm{n}=12-13)$. ${ }^{*} \mathrm{P}<0.05$ compared with all experimental groups (Mann-Whitney test). ${ }^{*} \mathrm{P}<0.001$ compared with all experimental groups (log-rank test).
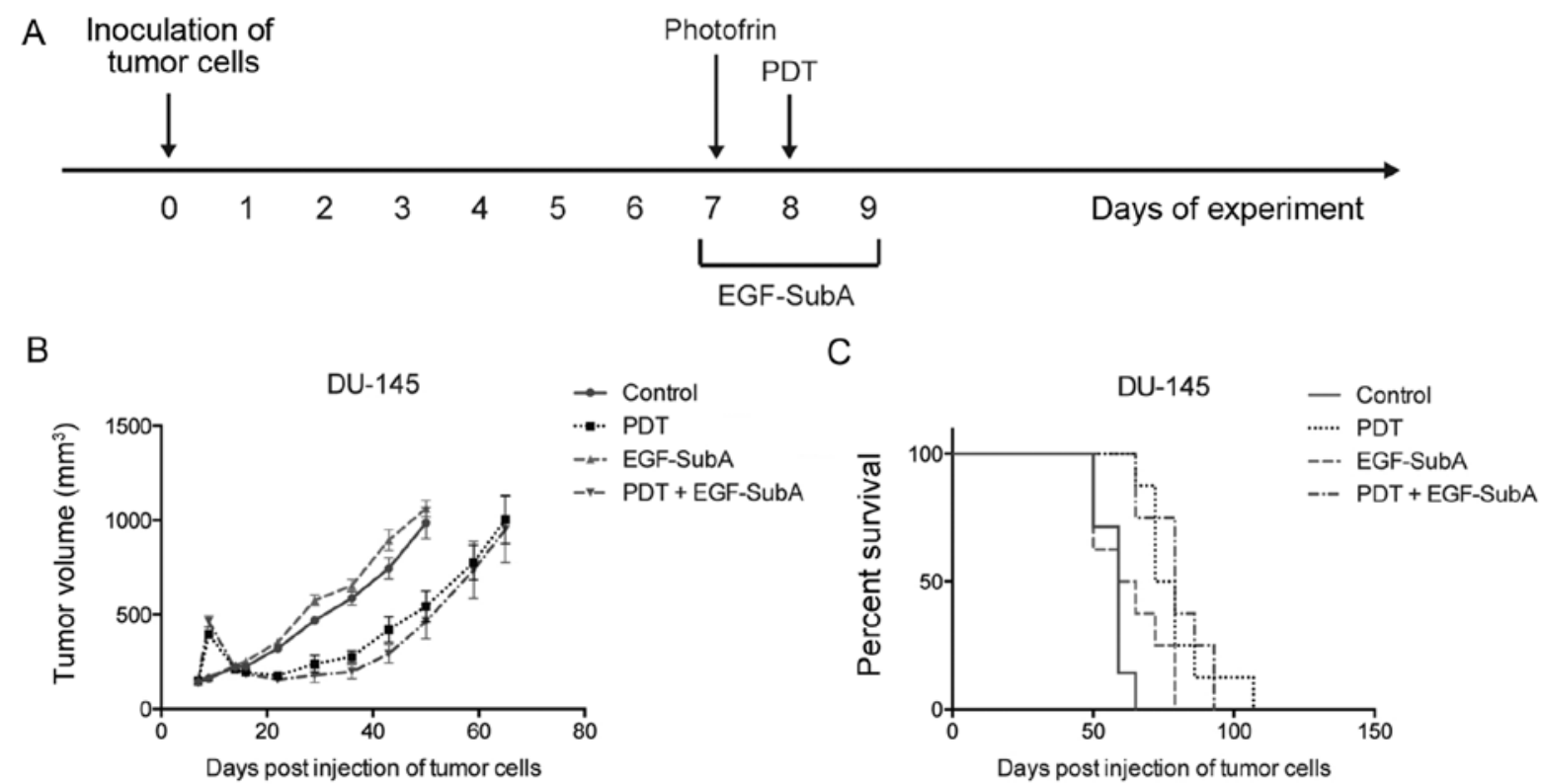

Figure 6. EGF-SubA + PDT treatment is ineffective in immunocompromised mice. (A) SCID mice were inoculated with 2x106 of DU-145 cells. On days 7-9, the mice were treated i.p with EGF-SubA $(25 \mu \mathrm{g} / \mathrm{kg})$ in PBS. Photofrin was administered i.p. at a dose of $10 \mathrm{mg} / \mathrm{kg}$ on day 7. Twenty-four hours later, the tumor site was illuminated with laser light at fluence of $43 \mathrm{~J} / \mathrm{cm}^{2}$. (B) Mean tumor volumes ( \pm SE). The experiment was performed once. (C) Kaplan-Meyer plots of the survival of the mice bearing DU-145 tumors $(n=7-8)$.

results are in line with previous findings that a combination of a high dose of cyclophosphamide, which decreases the number of splenocytes, reduced the survival of mice and impaired PDT efficacy (25). Collectively these results imply that a disturbance of the immune response may have detrimental impact on the overall PDT effects and may have important implications for the evaluation of novel agents in combination with PDT.
Another important finding was that EGF-SubA cytotoxin at sub-lethal doses affects the immune system. EGF-SubA is a fusion protein composed of EGF and a catalytic subunit of the holotoxin SubAB5, and was designed to selectively target EGFR-overexpressing tumor cells. Backer et al (18) demonstrated that the EGF-SubA fusion protein selectively kills tumor cells expressing EGFR and suppresses the growth of 
these tumors in vivo at a dose of $125 \mu \mathrm{g} / \mathrm{kg}$, without observable toxic effects. In our experimental settings, doses of EGF-SubA exceeding $100 \mu \mathrm{g} / \mathrm{kg}$ were already toxic, leading to the deaths of approximately half of the treated animals after two administrations of the fusion protein (data not shown). The difference may result from various dosing schedules. The lower EGF-SubA dose, $50 \mu \mathrm{g} / \mathrm{kg}$, did not trigger any lethal effects, yet it decreased the numbers of immune cells in the spleens of treated animals (Fig. 2). Moreover, the histopathological examinations of the livers of mice treated with $50 \mu \mathrm{g} /$ $\mathrm{kg}$ EGF-SubA revealed the presence of necrotic and hemorrhagic foci (Fig. 3). These observations are reminiscent of the effects reported in mice treated with SubAB5 holotoxin (16). Several possible explanations exist as to how the fusion protein affects cells not expressing EGFR. It may be transmitted to immune cells via endocytosis together with dying tumor cells. Alternatively, the EGF-SubA fusion protein tropism may not be restricted to EGFR-expressing cells, especially when administered at higher doses.

In summary, we showed that GRP78-targeting EGF-SubA cytotoxin improves the efficacy of PDT in vivo, but only at a lower dose which does not impair the immune system. Our results further substantiate the indispensable role of the adaptive immunity in achieving long-term curative PDT effects.

\section{Acknowledgements}

We thank Ewa Werner, Karolina Hajduk, Anna Czerepinska and Elzbieta Gutowska for valuable technical assistance. This study was supported by grants: DI2011 021141 (M.G.) and DI2013 006643 (A.D.) from Polish Ministry of Science and Higher Education and FP7-REGPOT-2012-CT2012-316254-BASTION from the European Commission 7th Framework Programme (J.G.).

\section{References}

1. Agostinis P, Berg K, Cengel KA, Foster TH, Girotti AW, Gollnick SO, Hahn SM, Hamblin MR, Juzeniene A, Kessel D, et al: Photodynamic therapy of cancer: An update. CA Cancer J Clin 61: 250-281, 2011.

2. Firczuk M, Nowis D and Gołab J: PDT-induced inflammatory and host responses. Photochem Photobiol Sci 10: 653-663, 2011.

3. Korbelik M, Krosl G, Krosl J and Dougherty GJ: The role of host lymphoid populations in the response of mouse EMT6 tumor to photodynamic therapy. Cancer Res 56: 5647-5652, 1996.

4. Korbelik M and Cecic I: Contribution of myeloid and lymphoid host cells to the curative outcome of mouse sarcoma treatment by photodynamic therapy. Cancer Lett 137: 91-98, 1999.

5. Garg AD, Krysko DV, Verfaillie T, Kaczmarek A, Ferreira GB, Marysael T, Rubio N, Firczuk M, Mathieu C, Roebroek AJ, et al: A novel pathway combining calreticulin exposure and ATP secretion in immunogenic cancer cell death. EMBO J 31: 1062-1079, 2012.

6. Korbelik M, Sun J and Cecic I: Photodynamic therapy-induced cell surface expression and release of heat shock proteins: Relevance for tumor response. Cancer Res 65: 1018-1026, 2005.

7. Henderson BW, Gollnick SO, Snyder JW, Busch TM, Kousis PC, Cheney RT and Morgan J: Choice of oxygen-conserving treatment regimen determines the inflammatory response and outcome of photodynamic therapy of tumors. Cancer Res 64: 2120-2126, 2004.

8. Kabingu E, Oseroff AR, Wilding GE and Gollnick SO: Enhanced systemic immune reactivity to a Basal cell carcinoma associated antigen following photodynamic therapy. Clin Cancer Res 15: 4460-4466, 2009.
9. Korbelik M and Cecic I: Enhancement of tumour response to photodynamic therapy by adjuvant mycobacterium cell-wall treatment. J Photochem Photobiol B 44: 151-158, 1998.

10. Wachowska M, Gabrysiak M, Muchowicz A, Bednarek W, Barankiewicz J, Rygiel T, Boon L, Mroz P, Hamblin MR and Golab J: 5-Aza-2'-deoxycytidine potentiates antitumour immune response induced by photodynamic therapy. Eur J Cancer 50: 1370-1381, 2014

11. Jalili A, Makowski M, Switaj T, Nowis D, Wilczynski GM, Wilczek E, Chorazy-Massalska M,Radzikowska A, Maslinski W, Biały L, et al: Effective photoimmunotherapy of murine colon carcinoma induced by the combination of photodynamic therapy and dendritic cells. Clin Cancer Res 10: 4498-4508, 2004.

12. Xue LY, Agarwal ML and Varnes ME: Elevation of GRP-78 and loss of HSP-70 following photodynamic treatment of V79 cells: Sensitization by nigericin. Photochem Photobiol 62: 135-143, 1995.

13. Luo B and Lee AS: The critical roles of endoplasmic reticulum chaperones and unfolded protein response in tumorigenesis and anticancer therapies. Oncogene 32: 805-818, 2013.

14. Paton AW, Beddoe T, Thorpe CM, Whisstock JC, Wilce MC, Rossjohn J, Talbot UM and Paton JC: AB5 subtilase cytotoxin inactivates the endoplasmic reticulum chaperone BiP. Nature 443: 548-552, 2006

15. Paton AW, Srimanote P, Talbot UM, Wang H and Paton JC: A new family of potent $\mathrm{AB}(5)$ cytotoxins produced by Shiga toxigenic Escherichia coli. J Exp Med 200: 35-46, 2004.

16. Wang H, Paton JC and Paton AW: Pathologic changes in mice induced by subtilase cytotoxin, a potent new Escherichia coli AB5 toxin that targets the endoplasmic reticulum. J Infect Dis 196: 1093-1101, 2007.

17. Harama D, Koyama K, Mukai M, Shimokawa N, Miyata M, Nakamura Y, Ohnuma Y, Ogawa H, Matsuoka S, Paton AW, et al: A subcytotoxic dose of subtilase cytotoxin prevents lipopolysaccharide-induced inflammatory responses, depending on its capacity to induce the unfolded protein response. J Immunol 183: 1368-1374, 2009.

18. Backer JM, Krivoshein AV, Hamby CV, Pizzonia J, Gilbert KS, Ray YS, Brand H, Paton AW, Paton JC and Backer MV: Chaperone-targeting cytotoxin and endoplasmic reticulum stress-inducing drug synergize to kill cancer cells. Neoplasia 11: 1165-1173, 2009.

19. Firczuk M, Gabrysiak M, Barankiewicz J, Domagala A, Nowis D, Kujawa M, Jankowska-Steifer E, Wachowska M, Glodkowska-Mrowka E, Korsak B, et al: GRP78-targeting subtilase cytotoxin sensitizes cancer cells to photodynamic therapy. Cell Death Dis 4: e741, 2013.

20. Greulich H, Chen TH, Feng W, Jänne PA, Alvarez JV, Zappaterra M, Bulmer SE, Frank DA, Hahn WC, Sellers WR, et al: Oncogenic transformation by inhibitor-sensitive and -resistant EGFR mutants. PLoS Med 2: e313, 2005.

21. Makowski M, Grzela T, Niderla J, ŁAzarczyk M, Mróz P, Kopeé M, Legat M, Strusińska K, Koziak K, Nowis D, et al: Inhibition of cyclooxygenase-2 indirectly potentiates antitumor effects of photodynamic therapy in mice. Clin Cancer Res 9: 5417-5422, 2003.

22. Szokalska A, Makowski M, Nowis D, Wilczynski GM, Kujawa M, Wójcik C, Mlynarczuk-Bialy I, Salwa P, Bil J, Janowska S, et al: Proteasome inhibition potentiates antitumor effects of photodynamic therapy in mice through induction of endoplasmic reticulum stress and unfolded protein response. Cancer Res 69: 4235-4243, 2009.

23. Korbelik M: Induction of tumor immunity by photodynamic therapy. J Clin Laser Med Surg 14: 329-334, 1996.

24. Kabingu E, Vaughan L, Owczarczak B, Ramsey KD and Gollnick SO: $\mathrm{CD}^{+} \mathrm{T}$ cell-mediated control of distant tumours following local photodynamic therapy is independent of CD4 ${ }^{+}$ $\mathrm{T}$ cells and dependent on natural killer cells. Br J Cancer 96: 1839-1848, 2007.

25. Castano AP, Mroz P, Wu MX and Hamblin MR: Photodynamic therapy plus low-dose cyclophosphamide generates antitumor immunity in a mouse model. Proc Natl Acad Sci USA 105: 5495-5500, 2008. 